University of Nebraska - Lincoln

DigitalCommons@University of Nebraska - Lincoln

Stephen Ducharme Publications

Research Papers in Physics and Astronomy

July 2001

\title{
General features of the intrinsic ferroelectric coercive field
}

V.M. Fridkin

University of Nebraska - Lincoln

Stephen Ducharme

University of Nebraska, sducharme1@unl.edu

Follow this and additional works at: https://digitalcommons.unl.edu/physicsducharme

Part of the Physics Commons

Fridkin, V.M. and Ducharme, Stephen, "General features of the intrinsic ferroelectric coercive field" (2001). Stephen Ducharme Publications. 12.

https://digitalcommons.unl.edu/physicsducharme/12

This Article is brought to you for free and open access by the Research Papers in Physics and Astronomy at DigitalCommons@University of Nebraska - Lincoln. It has been accepted for inclusion in Stephen Ducharme Publications by an authorized administrator of DigitalCommons@University of Nebraska - Lincoln. 


\title{
MAGNETISM \\ AND FERROELECTRICITY
}

\section{General Features of the Intrinsic Ferroelectric Coercive Field}

\author{
V. M. Fridkin ${ }^{1,2}$ and S. Ducharme ${ }^{1 *}$ \\ ${ }^{1}$ Department of Physics and Astronomy, Center for Material Research and Analysis University of Nebraska, \\ 68588-0111 Lincoln, USA \\ e-mail: sducharme1@unl.edu \\ ${ }^{2}$ Shubnikov Institute of Crystallography, Russian Academy of Sciences, Leninskiu pr. 59, Moscow, 117333 Russia \\ e-mail:fridkin@ns.crys.ras.ru \\ Received December 14, 2000
}

\begin{abstract}
The value of the intrinsic ferroelectric coercive field is obtained independently, from general energy considerations and from the predictions of several models of the ferroelectric state. All predictions yield a value of the order of the depolarization field, which is equal to the spontaneous polarization divided by the dielectric permittivity, and are consistent with the recent measurements of the intrinsic ferroelectric coercive field in ultrathin Langmuir-Blodgett films of copolymers of polyvinylidene fluoride with trifluoroethylene. Prior studies succeeded only in measuring the much smaller extrinsic coercive fields, which are limited by nucleation processes and domain motion. (C) 2001 MAIK "Nauka/Interperiodica".
\end{abstract}

The characteristic feature of a ferroelectric is the ability of its polarization to be reversed or switched repeatably through the application of a sufficiently large external electric field, as illustrated by the hysteresis loops in Fig. 1. However, since the discovery of ferroelectricity 80 years ago by Valasek, few published reports have considered the value of the intrinsic coercive field connected with collective polarization reversal, a consequence of the instability of the macroscopic polarization state in an opposing electric field. This is because the measured value of the ferroelectric coercive field was invariably much smaller than the intrinsic value predicted by theory, such as the Landau-Ginzburg (LG) mean-field model [1] and the ferroelectric Ising model $[2,3]$. The low extrinsic coercive field observed in real ferroelectric crystals and films is caused by nucleation of domains with reversed polarization, which then grow and coalesce by domain wall motion $[2,4,5]$. Nucleation can occur at fixed defects in the crystal or due to a passive (nonferroelectric) surface layer [6]. Nucleation mechanisms and domain wall dynamics must be arbitrarily inserted into these models in order to explain the experimental observations of switching kinetics with much lower coercive fields in real ferroelectric materials [2]. In fact, the study and modeling of extrinsic switching is of great importance and is central to the application of ferroelectric films to nonvolatile random-access memories [7].

It has proven difficult to prevent extrinsic switching by eliminating defects or by pinning domain walls. Another way to achieve intrinsic switching is to make a particle small enough or a film thin enough to inhibit nucleation [5, 8-10]. Several mechanisms for this

\footnotetext{
* This article was submitted by the authors in English.
}

finite-size effect have been proposed, including reduction of the nucleation volume, introduction of a space charge near the electrodes, elimination of passive layers, and domain-wall pinning $[4,6,10,11]$. Even in the thinnest ferroelectric films obtained previously, the measured extrinsic coercive field is much smaller than the expected intrinsic value calculated from theoretical models, and, consequently, there has been relatively little published discussion of intrinsic switching, except to note that it remained out of reach. Thin ferroelectric

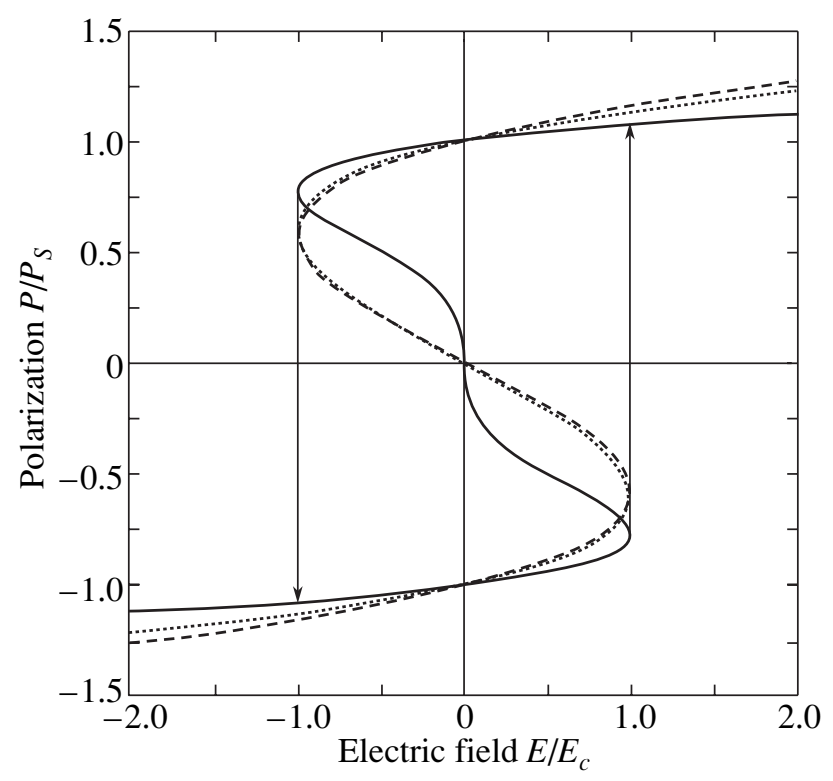

Fig. 1. Theoretical ferroelectric hysteresis loops from the first-order Landau-Ginzburg model (solid line), secondorder Landau-Ginzburg model (dashed line), and IsingDevonshire model (dotted line). 
films have indeed shown the nucleation-inhibition finite-size effect, an increase in the coercive field with a decrease in thickness, but the value of the measured coercive field was still much lower than the intrinsic one, and, correspondingly, the switching was still controlled by nucleation and domains. Recently [12], we succeeded in finding the limiting intrinsic coercive field in films as thin as $1 \mathrm{~nm}$ made by Langmuir-Blodgett (LB) deposition of a two-dimensional ferroelectric copolymer [13].

The measured values of the intrinsic coercive field were in good agreement with the predictions of the LG mean-field theory $[1,14]$. Here, we analyze the calculation of the intrinsic coercive field from a more general perspective and from several specific models of ferroelectricity.

We can estimate the intrinsic ferroelectric coercive field $E_{c}$ by calculating the external electric field necessary to overcome the depolarization field $P_{S} / \varepsilon \varepsilon_{0}$, where $P_{S}$ is the spontaneous polarization, $\varepsilon$ is the dielectric constant (more precisely, the contribution to the dielectric constant from ferroelectricity; the total dielectric constant of the medium $\varepsilon_{T}=\varepsilon_{B}+\varepsilon$ contains contributions from the background polarizability and the ferroelectric polarizability) along the direction of spontaneous polarization, and $\varepsilon_{0}$ is the permittivity of free space. The energy density associated with the spontaneous polarization is $u_{P}=(1 / 2) P_{S}^{2} / \varepsilon \varepsilon_{0}$. Application of an external field $E$ in a direction opposite to the spontaneous polarization would contribute to the energy density $u_{E}=\mathbf{E} \cdot \mathbf{P}_{S}=-E P_{S}{ }^{1}$ The intrinsic coercive field $E_{c}$ is then approximately the value of the applied field that produces an energy comparable to the polarization energy, $u_{E} \approx u_{P}$, so that the intrinsic coercive field is approximately half the depolarization field:

$$
E_{c} \approx \frac{1}{2} \frac{P_{S}}{\varepsilon \varepsilon_{0}} \quad \text { (from energy). }
$$

The intrinsic coercive field for a continuous ferroelectric-paraelectric first-order (close to second-order) phase transition can be calculated from the LandauGinzburg phenomenology (LG1) $[15,16]$. The LG1 Gibbs free energy of a uniaxial material is $G=G_{0}+$ $(1 / 2) \alpha P^{2}+(1 / 4) \beta P^{4}+(1 / 6) \gamma P^{6}-P E$, where the first coefficient has the Curie-Weiss form $\alpha=\left(T-T_{0}\right) /\left(\varepsilon_{0} C\right)$, $T_{0}$ is the Curie temperature, $C$ is the Curie constant, and the second coefficient obeys the condition $\beta<0$, whereas the condition for the necessary third coefficient is $\gamma>0$. The parameters $C, T_{0}, \beta$, and $\gamma$ are assumed independent of temperature and the electric

\footnotetext{
${ }^{1}$ This contribution applies to all kinds of ferroelectrics, whether displacive, order-disorder, or other. For example, in ferroelectric materials consisting of fixed dipoles with the dipole moment $\mu$ and density $N$, as in ferroelectric polymers, the macroscopic polarization is $\mathbf{P}_{S}=-N\langle\boldsymbol{\mu}\rangle$ and the energy density of the dipoles in the applied field is $u_{E}=-N\langle\mathbf{E} \cdot \boldsymbol{\mu}\rangle$, which leads to the same result.
}

field. From the minimum of the free energy density, it is possible to calculate the properties of the ferroelectric phase that exists just below the zero-field transition temperature $T_{C}=T_{0}+(3 / 16) \varepsilon_{0} C \beta^{2} / \gamma$. In the ferroelectric phase, the spontaneous polarization is $P_{S}=$ $\pm \sqrt{-\beta(1+\sqrt{1-t}) / 2 \gamma}$, where $t=4 \alpha \gamma / \beta^{2}$ and the ferroelectric contribution to the dielectric constant is given by $1 / \varepsilon \varepsilon_{0}=-8\left(T-T_{C}\right) / C \varepsilon_{0}+(9 / 4)\left(\beta^{2} / \gamma\right)$. The LG1 intrinsic coercive field in the temperature range from just below $T_{0}(t=0)$ up to $T_{C}(t=3 / 4)$ is closely approximated by $E_{c} \approx 2 \beta(3|\beta| / 5 \gamma)^{3 / 2}(1-(25 / 24) t)[12,17]$, so the coercive field can be written as

$$
E_{c} \approx \frac{3 \sqrt{3}}{25 \sqrt{5}} \frac{P_{S}}{\varepsilon \varepsilon_{0}}\left(1-\frac{1}{6} t\right) \quad \text { (from LG1). }
$$

The value of the intrinsic coercive field $E_{c}$ near the Curie temperature $T_{0}$ is about one-tenth of the depolarization field.

The Landau-Ginzburg continuous ferroelectric phase transition of the second order (LG2) [1] is modeled with a free energy $G=G_{0}+(1 / 2) \alpha P^{2}+(1 / 4) \beta P^{4}-$ $P E$, where the first coefficient is again $\alpha=(T-$ $\left.T_{0}\right) /\left(\varepsilon_{0} C\right)$, and the second coefficient $\beta>0$. The ferroelectric phase in a zero electric field exists below the transition temperature $T_{C}=T_{0}$, where the spontaneous polarization $P_{S}= \pm \sqrt{-\alpha / \beta}$ and the ferroelectric contribution to the dielectric constant is given by $1 / \varepsilon \varepsilon_{0}=-2 \alpha$. The intrinsic coercive field $E_{c}=2 \beta(-\alpha / 3 \beta)^{3 / 2}$ as calculated from the LG2 free energy can be written [17]

$$
E_{c}=\frac{1}{3 \sqrt{3}} \frac{P_{S}}{\varepsilon \varepsilon_{0}} \quad \text { (from LG2). }
$$

Devonshire [16] applied the Ising model to ferroelectrics consisting of electric dipoles with dipole moment $p_{0}$, which can achieve a collective ordered state. The two-level (spin-1/2) Ising-Devonshire (ID) model describes a uniaxial ferroelectric that undergoes an order-disorder phase transition with the order parameter equal to the normalized spontaneous polarization $S=P_{S} / P_{S 0}$, where $P_{S 0}=N p_{0}$. The order parameter is obtained from the transcendental equation $[2,3,16]$ $S=\tanh \left[\left(J_{0} S+E p_{0}\right) / k T\right]$, where $k$ is the Boltzmann constant and $J_{0}$ is the pseudospin Ising interaction constant. In the ferroelectric phase near (but below) $T_{C}=T_{0}=J_{0} / k$, the zero-field spontaneous polarization follows a simple $1 / 2$ power scaling $S \approx \pm \sqrt{3\left(1-T / T_{C}\right)}$ and the dielectric constant is given by $1 / \varepsilon \varepsilon_{0} \approx 2\left[\left(k T_{0}\right) /\left(N p_{0}^{2}\right)\right]\left[1-T / T_{0}\right]$. The ID intrinsic coercive field near $T_{C}$ is approximately 
Typical experimental values of the coercive field $E_{c}^{\mathrm{exp}}$ compared to the depolarization field $E_{d}$ for $\mathrm{BaTiO}_{3}$, TGS, and BL copolymer films (all values were obtained near $T_{0}$ )

\begin{tabular}{l|c|c|c|c|c}
\hline \multicolumn{1}{c|}{ Material } & $\varepsilon$ & $P_{S}, \mathrm{C} / \mathrm{m}^{2}$ & $E_{c}^{\exp }, \mathrm{MV} / \mathrm{m}$ & $\begin{array}{c}E_{d}=P_{S} / \varepsilon \varepsilon_{0}, \\
\mathrm{MV} / \mathrm{m}\end{array}$ & $E_{c}^{\exp } /(1 / 2) E_{d}$ \\
\hline $\mathrm{BaTiO}_{3}[18]$ & 150 & 0.26 & 0.20 & 196 & 0.0020 \\
Triglycine sulphate (TGS) [19] & 43 & 0.028 & 0.011 & 74 & 0.00030 \\
$\mathrm{KD}_{2} \mathrm{PO}_{4}[20]$ & 43 & 0.062 & 0.34 & 163 & 0.0042 \\
$\mathrm{PZr}_{0.25} \mathrm{Ti}_{0.75} \mathrm{O}_{3}$ (PZT) 100 nm thin film [21] & 200 & 0.38 & 10 & 215 & 0.093 \\
Polyvinylidene fluoride (PVDF) [22] & 11 & 0.065 & 55 & 667 & 0.16 \\
$P(\mathrm{VDF}:$ TrFE 65 : 35) [23] & 9.5 & 0.090 & 45 & 1070 & 0.084 \\
$P(\mathrm{VDF}: \operatorname{TrFE~75~:~25)~60~nm~thin~film~[24]~}$ & 10 & 0.10 & 125 & 1129 & 0.22 \\
$P($ VDF-TrFE 70 : 30) $\leq 15 \mathrm{~nm}$ thin film [13, 17] & 8 & 0.10 & 480 & 1412 & 0.68 \\
\hline
\end{tabular}

Ratio $E_{c} /(1 / 2) E_{d}\left(\right.$ at $\left.T_{0}\right)$ predicted by model

\begin{tabular}{l|l} 
LG1 & 0.18 \\
LG2 & 0.38 \\
ID & 0.38
\end{tabular}

$E_{c} \approx\left[\left(2 k T_{0}\right) /\left(3 p_{0}\right)\right]\left[1-T / T_{0}\right]^{3 / 2}$, so the coercive field near $T_{C}$ can be written as

$$
E_{c} \approx \frac{1}{3 \sqrt{3}} \frac{P_{S}}{\varepsilon \varepsilon_{0}} \quad(\mathrm{ID}) \text {. }
$$

Note that the ID and LG2 models are equivalent near $T_{0}$ and predict the same temperature dependences for the spontaneous polarization, dielectric constant, and coercive field, because near $T_{C}$, the ID order parameter $S$ is small and the free energy of the ID model can be expanded in powers of $P_{S}$ to reproduce the LG2 free energy.

The value of the coercive field near the Curie temperature $T_{0}$ obtained from the three mean-field models (Eqs. 2-4) ranges from 10 to $20 \%$ of the depolarization

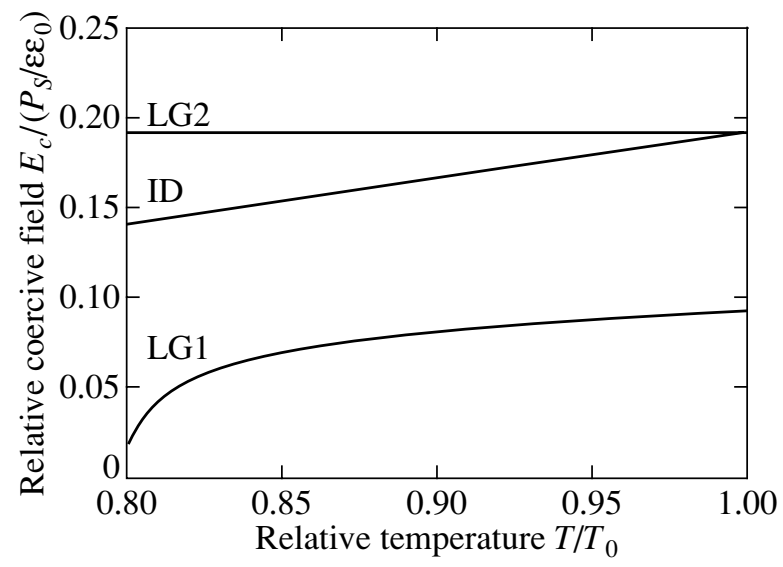

Fig. 2. Intrinsic coercive field $E_{c}$ normalized to the depolarization field $P_{S} / \varepsilon \varepsilon_{0}$ calculated from the LG2 model, LG1 model, and ID model. field $P_{S} / \varepsilon \varepsilon_{0}$, a little less than the value of $50 \%$ estimated from basic energy considerations (Eq. 1), even as the spontaneous polarization and dielectric constant vary strongly with temperature. The predictions hold well near $T_{0}$ for all models, as shown in Fig. 2. Therefore, the essential existence and approximate value of the intrinsic coercive field does not depend on the nature of the ferroelectric transition, whether it is first order or second order, displacive or order-disorder, from permanent dipoles or induced dipoles. However, the measured dielectric constant includes the background contribution and, near $T_{C}$, the ferroelectric contribution is typically larger than the equilibrium value because of thermal hysteresis, particularly in ferroelectrics with a first-order phase transition. Therefore, the value of the depolarization field $P_{S} / \varepsilon \varepsilon_{0}$ obtained from direct measurements near $T_{C}$ will likely be somewhat less than the intrinsic value.

Measurements of the coercive field in bulk ferroelectric crystals have invariably yielded values much smaller than the intrinsic value, as summarized in the table. While the traditional ferroelectrics have extremely low coercive fields, the ferroelectric polymer polyvinylidene fluoride (PVDF) and its copolymers show considerably higher coercive fields, typically $50 \mathrm{MV} / \mathrm{m}$ in "bulk" films, approaching the intrinsic value predicted by various models. By 1986, Kimura and Ohigashi [24] reported that copolymer films thinner than $1 \mu \mathrm{m}$ showed increasing coercive fields with decreasing thickness (Fig. 3) up to a then-record coercive field of $125 \mathrm{MV} / \mathrm{m}$ in a copolymer film $60 \mathrm{~nm}$ thick [24]. But the solvent-spinning techniques used would not yield thinner films of sufficient quality and dielectric strength, so the measurement of the intrinsic coercive field lay just beyond reach. 


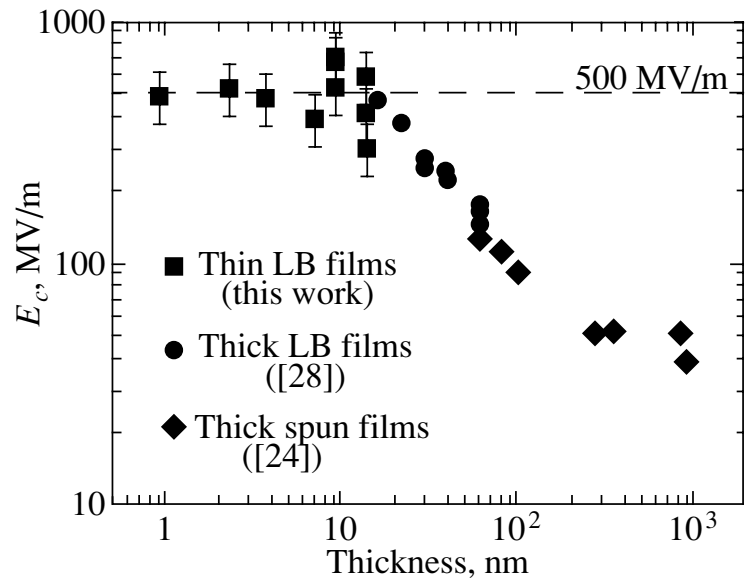

Fig. 3. Scaling of the coercive field with film thickness, measured at or near $25^{\circ} \mathrm{C}$, from ferroelectric vinylidene fluoride-trifluoroethylene copolymer films. Adapted from [12].

The successful fabrication by LB deposition of ultrathin films of PVDF and copolymers with trifluoroethylene (TrFE) beginning in 1995 [25-27] presented us with highly crystalline films with polymer chains lying in the film plane and with the polarization axis perpendicular to the film. These films, ranging in thickness from $1 \mathrm{~nm}$ to over $100 \mathrm{~nm}$, allowed us to study ferroelectricity down to the monolayer level [12, 13, 25-30]. One of the surprising results of these studies was the discovery of two-dimensional ferroelectricity [13], as the ferroelectric phase persisted in the thinnest films, with no significant decrease in the transition temperature, contrary to the common expectation that finite-size scaling would suppress ferroelectricity. But finite-size effects did appear to suppress nucleation in films thinner than $1 \mu \mathrm{m}[24,28]$. As Fig. 3 shows, the rising coercive field with decreasing thickness $d$ observed by Kimura and Ohigashi continues with the thinner LB films to follow a $d^{-0.7}$ power law scaling down to a thickness of $15 \mathrm{~nm}$ [28], consistent with a finite-thickness suppression of nucleation or domain wall motion.

The films of a thickness of $15 \mathrm{~nm}$ (30 monolayers) or less, on the other hand, had coercive fields of about $500 \mathrm{MV} / \mathrm{m}$ at $25^{\circ} \mathrm{C}$, which is comparable to the theoretical intrinsic value [12] calculated with the LG1 model with free-energy coefficients determined in prior studies $[30,31]$. Because of uncertainties in the LG1 parameters, the good coincidence between the measured and expected coercive fields did not ensure that it was indeed the intrinsic value. However, cessation of finite-size scaling below $15 \mathrm{~nm}$ implies that polarization reversal is no longer initiated by nucleation; that is, nucleation is inhibited and polarization reversal depends only on the intrinsic ferroelectric properties. Detailed measurements of the temperature dependence of the coercive field confirmed the specific predictions of the LG1 model [12] and thus established that the measurements coincided with the intrinsic coercive field. Though nucleation and domains do not limit the value of the coercive field, they could play a key role in switching dynamics. Preliminary imaging of the polarization through pyroelectric scanning microscopy has not revealed domains larger than the $2 \mu \mathrm{m}$ resolution of our current system, but further study is necessary before something can be said about domain and crystallite properties.

It is not clear whether or not the two-dimensional nature of ferroelectricity in the vinylidene fluoride copolymers was essential for this achievement to be made. There may soon be results bearing on this question as several groups have reported good films with thicknesses less than $10 \mathrm{~nm}$ made from several known bulk ferroelectric materials [21, 32-34]. Recent improvements in the quality of solvent-crystallized vinylidene fluoride copolymers should help elucidate the effects of nucleation and domain-wall motion in bulk crystals [35-37].

\section{ACKNOWLEDGMENTS}

This work was supported by the USA National Science Foundation and the Nebraska Research Initiative.

\section{REFERENCES}

1. V. L. Ginzburg, Zh. Éksp. Teor. Fiz. 15, 739 (1945).

2. M. E. Lines and A. M. Glass, Principles and Applications of Ferroelectrics and Related Materials (Clarendon, Oxford, 1977; Mir, Moscow, 1981).

3. B. A. Strukov and A. P. Levanuk, Ferroelectric Phenomena in Crystals (Nauka, Moscow, 1995; Springer-Verlag, Berlin, 1998).

4. W. J. Merz, Phys. Rev. 95, 690 (1954).

5. W. J. Merz, J. Appl. Phys. 27, 938 (1956).

6. A. M. Bratkovsky and A. P. Levanyuk, Phys. Rev. Lett. 84, 3177 (2000).

7. O. Auciello, J. F. Scott, and R. Ramesh, Phys. Today 51, 22 (1998).

8. K. Dimmler, M. Parris, D. Butler, et al., J. Appl. Phys. 61, 5467 (1987).

9. J. F. Scott, Phase Transit. 30, 107 (1991).

10. Y. Ishibashi, in Ferroelectric Thin Films: Synthesis and Basic Properties, Ed. by C. Paz de Araujo, J. F. Scott, and G. W. Taylor (Gordon and Breach, Amsterdam, 1996), p. 135.

11. A. K. Tagantsev, Integr. Ferroelectr. 16, 237 (1997).

12. S. Ducharme, V. M. Fridkin, A. V. Bune, et al., Phys. Rev. Lett. 84, 175 (2000).

13. A. V. Bune, V. M. Fridkin, S. Ducharme, et al., Nature 391, 874 (1998).

14. V. L. Ginzburg, J. Phys. USSR 10, 107 (1946).

15. V. L. Ginzburg, Zh. Éksp. Teor. Fiz. 19, 39 (1949).

16. A. F. Devonshire, Adv. Phys. 3, 85 (1954).

17. L. M. Blinov, V. M. Fridkin, S. P. Palto, et al., Usp. Fiz. Nauk 170, 247 (2000).

18. F. Jona and G. Shirane, Ferroelectric Crystals (Macmillan, New York, 1962; Mir, Moscow, 1965).

19. P. J. Lock, Appl. Phys. Lett. 19, 390 (1971). 
20. G. A. Samara, Ferroelectrics 5, 25 (1973).

21. T. Maruyama, M. Saitoh, I. Sakai, et al., Appl. Phys. Lett. 73, 3524 (1998).

22. M. A. Marcus, Ferroelectrics 40, 29 (1982).

23. T. Fukukawa, Ferroelectrics 57, 63 (1984).

24. K. Kimura and H. Ohigashi, Jpn. J. Appl. Phys. 25, 383 (1986).

25. S. Palto, L. Blinov, A. Bune, et al., Ferroelectr. Lett. Sect. 19, 65 (1995).

26. A. Bune, S. Ducharme, V. M. Fridkin, et al., Appl. Phys. Lett. 67, 3975 (1995).

27. S. Palto, L. Blinov, E. Dubovik, et al., Europhys. Lett. 34, 465 (1996).

28. L. M. Blinov, V. M. Fridkin, S. P. Palto, et al., Thin Solid Films 284-285, 474 (1996).

29. S. Ducharme, S. P. Palto, L. M. Blinov, and V. M. Fridkin, in Proceedings of the 6th Williamsburg Workshop on Fundamental Physics of Ferroelectrics, Ed. by R. Cohen and K. Rabe (American Inst. of Physics, Melville, 2000), p. 354.

30. S. Ducharme, A. V. Bune, V. M. Fridkin, et al., Phys. Rev. B 57, 25 (1998).

31. A. V. Bune, C. Zhu, S. Ducharme, et al., J. Appl. Phys. 85, 7869 (1999).

32. T. Tybell, C. H. Ahn, and J.-M. Triscone, Appl. Phys. Lett. 75, 856 (1999).

33. E. D. Specht, H.-M. Christen, D. P. Norton, and L. A. Boatner, Phys. Rev. Lett. 80, 4317 (1998).

34. J. F. M. Cillessen, M. W. J. Prins, and R. M. Wolf, J. Appl. Phys. 81, 2777 (1997).

35. H. Ohigashi, K. Omote, H. Abe, and K. Koga, Jpn. J. Appl. Phys. 68, 1824 (1999).

36. H. Ohigashi, K. Omote, and T. Gomyo, Appl. Phys. Lett. 66, 3281 (1995).

37. M. Hikosaka, K. Sakurai, H. Ohigashi, and T. Koizumi, Jpn. J. Appl. Phys. 32, 2029 (1993). 